\title{
What Are the Chances? fMRI Correlates of Observing High and Low-Probability Actions
}

\author{
Roger Newman-Norlund ${ }^{1}$, Kim Bruggink², Raymond Cuijpers ${ }^{3}$, Harold Bekkering ${ }^{2}$ \\ ${ }^{1}$ Department of Exercise Science, University of South Carolina, Columbia, USA \\ ${ }^{2}$ Donders Institute for Brain, Cognition and Behavior, Radboud University Nijmegen, Nijmegen, The Netherlands \\ ${ }^{3}$ Industrial Engineering \& Innovation Sciences, Human Technology Interaction Group, \\ Eindhoven University of Technology, Eindhoven, The Netherlands \\ Email: rnorlund@mailbox.sc.edu
}

Received October 5, 2012; revised November 7, 2012; accepted November 15, 2012

\begin{abstract}
Cognitive scientists often use probabilistic equations to model human behavior in ambiguous situations. How, where, and even if such probabilities are represented in the human brain remains largely unknown. Here, we manipulated the probability of simple bottle-pouring action based on two considerations, the relative fullness of two glasses and the relative distance between the two glasses and the bottle. Whole brain functional magnetic resonance imaging was used to measure brain activity while participants viewed probable and improbable pouring actions. Improbable actions elicited increased activity in the theory of mind (ToM) network, commonly found active when trying to grasp the intentions of others, whereas probable actions elicited increased activity in the human mirror neuron system (hMNS) and areas associated with mental imagery and memory. These data provide novel insight into the brain mechanisms humans use to distinguish between high and low-probability actions.
\end{abstract}

Keywords: Probability; fMRI; Human Mirror Neuron System; Action Observation

\section{Introduction}

The ability to make predictions about the future state of the world when placed in ambiguous situations is a fundamental and very useful capacity present in humans. Athletes predict the actions of their opponents, mothers make predictions regarding their baby's needs, and writers predict the responses of their reviewers. Cognitive scientists have long relied on probabilistic models to explain a wide variety of complex human behaviors including decision making, action planning, motor learning and behavior [1-5]. Probabilistic models are especially apt at making predictions in ambiguous situations like those mentioned above. In such models, events are typically assigned a specific likelihood after taking into account the current situation and prior experience according to a well known statistical approach known as empirical Bayes. While significant research has been conducted with regards to the neural correlates of probabilities as they relate to topics such as reinforcement learning, risk taking behavior and reward [6-9], relatively little is known about the neural representation of action probabilities as they relate to object-directed actions encountered in everyday situations. In the current experiment, we attempted to localize brain areas responsible for coding the probability of actions. Specifically, we con- ducted a functional magnetic resonance imaging (fMRI) experiment in which we could visualize brain activity during the observation of actions that were either likely or unlikely based on the relative distance between objects being combined or the context in which the combination of objects occurred.

Based on previous research, we had strong reason to believe that human mirror neuron system (MNS) would be involved in the calculation of action probabilities. The MNS, a system comprised of the human inferior parietal and frontal lobes, is often cited as supporting various aspects of action understanding. Activity within this system is modulated by the type of action sequences that are "likely" to follow a particular observed movement [1013]. For example, Fogassi and colleagues [14] found that activity recorded from primate MNs during the grasp of a peanut depends critically on whether the peanut was then eaten or placed in a cup. In humans, Iacoboni and colleagues [15] showed that activity recorded from the human MNS during the observation of a grasping action also varies as a function of the subsequent to-be performed movements. This sensitivity to future actions, highly relevant to the discussion of action probabilities, has been referred to as "action forecasting". Here, the idea is that MNs respond most strongly to actions which 
are likely to be followed by other actions, i.e. actions that are the initial element of a likely-to-follow action sequence.

The idea that mirror neuron activity may reflect the probability of a given action is echoed by researchers working in the field of biological robotics. These researchers have gone so far as to suggest specific biological mechanisms whereby action probabilities could be cortically represented. Specifically, Metta and colleagues [16] have hypothesized that the probability of a given action $A$ occurring, given the presence of a given object $O$, or $\mathrm{P}$ $A \mid O$, might be encoded by the response of canonical neurons located within core MNS areas. These neurons respond to the observation of objects that can be grasped, and it has been suggested that their activity reflects 1) the processing of the affordances of the object and 2) a "mental simulation" of actual object use and/or 3) subsequent actions that might possibly be executed [17].

In order to examine the anatomical basis of probability coding, we created a paradigm in which the probability of a simple pouring action (i.e. pouring a bottle of wine into one of two glasses) was dependent on two factors; the relative fullness of the two glasses which could potentially be poured into and the relative distance between the bottle and each of the two glasses. We arrived at these factors based on the computational model of Cuijpers and colleagues [2] which predicts that 1) the combination of nearby objects is more likely than the combination of distant objects, and that 2) logical combinations of objects (e.g. bolt + nut) are more probable than illogical combinations (e.g. bolt + screw). Accordingly, we hypothesized that the emptier a glass was, the more likely it would be for an actor to pour fluid into that glass. Similarly, we reasoned that the closer the bottle was to one of the glasses, the more likely it would be to pour into that glass. We expected the MNS to exhibit differential response patterns based on the probability of pouring actions which we experimentally manipulated by varying these distance and fullness cues. Specifically, we predicted the existence of sites within the MNS that would respond maximally to high-probability actions independent of whether the action's probability was based distance or fullness cues.

\section{Methods}

\subsection{Participants}

Twenty-one right-handed subjects ( 5 males, 16 females) between the ages of 19 and 35 (mean \pm SD age, $23.6 \pm$ 4.1 years) participated in the experiment. All participants had normal or corrected-to-normal vision and were healthy adults (self-report). They gave written informed consent according to the institutional guidelines set forth by the local ethics committee (CMO region Arnhem-Nijme- gen, Netherlands) prior to the experiment. Subjects were compensated at the rate of $12.50 € / \mathrm{hr}$ for their participation.

\subsection{Stimuli}

Stimuli for the scanning session consisted of photos presented centrally on the screen on a plain black background. Photos were made using a digital camera and resized to a $600 \times 400$ pixels image. Photos displayed a table with two glasses positioned on the left and right side of the table, a bottle and a person sitting behind the table (without showing the head). Photos were taken in such a way that the displayed person appeared to sit across the table facing the subject. Four types of stimuli can be distinguished. The first type of stimuli displayed the bottle on the table in between two wineglasses while the person is holding it with her/his right hand. Bottle position varied from left to right in five positions (closest to left glass, left from the middle, middle, right from the middle, closest to right glass) and two different varieties of wine bottle were used in an attempt to maintain subjects' attention. The second type of stimuli showed the person pouring from a bottle into either one of the glasses. Photos of glasses containing varying amounts of liquid (almost empty glasses, half full glasses and full glasses) were later overlayed on top of the glasses in the photos with the total setup. All 90 combinations were included $(5$ bottle positions $\times 2$ possibilities for pouring $\times 3$ fullness degrees for left glass $\times 3$ fullness degrees for right glass $=90$ possibilities).

\subsection{Testing Procedure}

Functional magnetic resonance imaging was performed while participants watched short, two-frame action sequences in which an actor gripped a wine bottle and poured it into one of two wine glasses. A single functional run consisted of 99 trials, nine of which were a repetition of the preceding trial. The other 90 trials were all different. The two different bottles were randomly distributed over the 90 possibilities mentioned above, and these combinations were presented in random order. Each trial consisted of a sequence of two photos interleaved with a fixation cross after every second photo. The first photo portrayed the initial set-up of the bottle and glasses and was presented for $2000 \mathrm{~ms}$. This was followed by a $1000 \mathrm{~ms}$ presentation of a photo showing the actor pouring wine into one of the glasses. Then a jitter stimulus containing a black screen with a white fixation cross in the middle was presented for 4000 - $8000 \mathrm{~ms}$. Figure 1 illustrates the temporal progression of the task. Stimuli were presented using a projector with a resolution of $1280 \times 1024$ pixels, and viewed by participants lying in the fMRI scanner through a custombuilt mirror. All sti- 

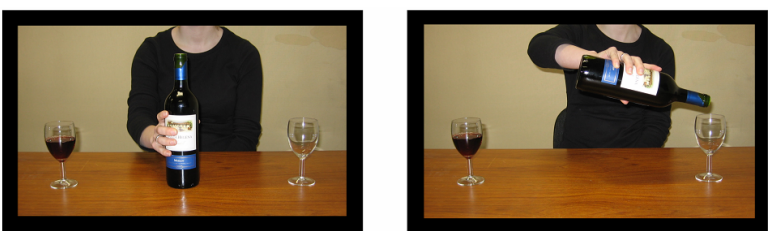

(a)
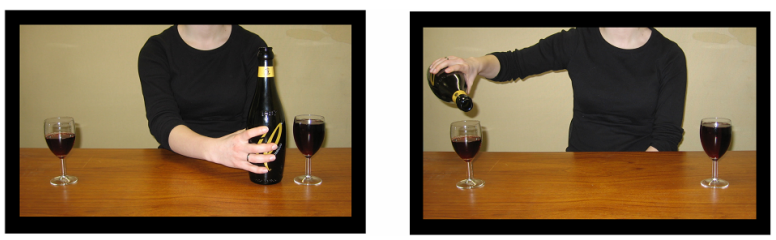

(b)

Figure 1. Examples of experimental stimuli. Plates showing example stimuli used in the experiment. (a) From left to right, sequence of pictures in which the probability of the action is equal based on the distance between the bottle and glasses. Based on the relative fullness of the glasses, it is more likely that the actor will pour into the right glass. (b) In this series of photos, it is more likely that the actor will pour into the right glass based on the distance between the bottle and glasses. Based on the relative fullness of the glasses, it is more probable that the actor will pour in the left glass.

muli were delivered using Presentation software version 9.90 (Neurobehavioral Systems, Davis, CA) run on a Dell Workstation (Austin, TX, USA). Subjects were instructed to concentrate on the photos while in the scanner and to respond with a button press when they observed the same trial two times in a row. This was done to ensure that participants were paying attention to the stimuli.

\section{4. fMRI Data Acquisition}

All magnetic resonance imaging was conducted at the F.C. Donders Centre for Cognitive Neuroimaging (Nijmegen, The Netherlands). Functional images were acquired on a Trio $3 \mathrm{~T}$ whole-body MR scanner (Siemens) using an ascending slice acquisition sequence and a birdcage head coil $\left(\mathrm{TR}=2.50 \mathrm{~s}, \mathrm{TE}=35 \mathrm{~ms}, 90^{\circ}\right.$ flip-angle, 34 axial slices, slice-matrix size $=64 \times 64$, slice thickness $=3 \mathrm{~mm}$, slice gap $=0.5 \mathrm{~mm}, \mathrm{FOV}=22.4 \mathrm{~cm}$, voxel size $=3.5 \times 3.5 \times 3.5 \mathrm{~mm})$. Head movement was restricted using foam cushions. A single scanning block lasted approximately 17 minutes. Following acquisition of echo-coplanar images (EPIs), a T1-weighted 3D MPRAGE sequence (volume TR $=1960 \mathrm{~ms}$, TE $=4.43 \mathrm{~ms}$, $8^{\circ}$ flip-angle, 176 coronal slices, slice-matrix size $=256$ $\times 208$, slice thickness $=1.0 \mathrm{~mm}$, voxel size $=1 \times 1 \times 1$ $\mathrm{mm}$ ) was acquired.

\section{5. fMRI Data Analysis}

Functional data were preprocessed and analyzed using SPM2 (http://www.fil.ion.ucllac.uk/spm). All functional data were first corrected for motion artifacts using the bilinear interpolation method and coregistered with the high resolution T2-weighted anatomical image. Images were then normalized to the Montreal Neurological Institute (MNI) template with a resolution of $2 \times 2 \times 2 \mathrm{~mm}$, and smoothed in three dimensions using a $6 \times 6 \times 6 \mathrm{~mm}$ Gaussian kernel. BOLD signal recorded during the observation of the short action sequences was modeled as the primary epoch of interest. Null events in which the fixation cross remained on the screen (as opposed to the appearance of an action sequence) were modeled as the REST condition along with the first and last fifty seconds of the functional run during which time the fixation cross was also on the screen.

\section{Results}

\subsection{One-Back Task}

In order to ensure that participants were paying attention to the stimuli, a small percentage of the trials (nine trials per functional fun) were replications of the immediately preceding trial. Participants pressed a response button as soon as they perceived one of these repeated trials. On average participants made 8.95 responses $(\mathrm{SD}=1.31$, $\operatorname{Min}=5, \operatorname{Max}=12$, Mode $=9$ ) during the experimental run thus confirming that they were paying attention to the stimuli.

\subsection{Brain Areas Responding to Low Probability Actions}

In order to isolate core brain areas responding more strongly to improbable as compared to probable actions we computed the intersection analysis $\left(\mathrm{LP}_{\mathrm{d}}-\mathrm{HP}_{\mathrm{d}}\right) \cap$ $\left(\mathrm{LP}_{\mathrm{f}}-\mathrm{HP}_{\mathrm{f}}\right)$ (see Methods). This analysis revealed that BOLD signal was significantly greater during the observation of low as compared to high-probability actions at sites in the left medial frontal cortex (MFC, BA 32) and the right middle superior temporal sulcus (mSTS, BA 48), (Table 1, Figure 2).

\subsection{Brain Areas Responding to High Probability Actions}

We conducted a separate intersection analysis to determine brain areas responding preferentially to the observation of high-probability actions based on either distance or fullness cues. Here, we calculated the conjunction $\left(\mathrm{HP}_{\mathrm{d}}-\mathrm{LP}_{\mathrm{d}}\right) \cap\left(\mathrm{HP}_{\mathrm{f}}-\mathrm{LP}_{\mathrm{f}}\right)$. This analysis revealed that BOLD signal was significantly greater during the observation of high as compared to low-probability actions at sites in the left SMG (BA 41 and 48), precuneus (BA 7), left superior frontal sulcus (BA 48) and bilateral visual/occipital cortex (BA 17,18,19) (Table 1, Figure 3). 
Table 1. Locations in MNI coordinates and labels of brain areas which responded preferentially to either high-probability actions or to and low-probability actions. Results for individual comparisons of high and low-probability actions based on distance and fullness were entered into an inclusive intersection analysis such that only areas surviving this threshold in both comparisons survived. In the case of High-probability Actions, results for the intersection of (HP_fullness - LP_fullness) $\cap$ (HP distance - LP distance) are shown. In the case of Low-probability Actions, the intersection of (LP fullness - HP fullness) $\cap$ (LP_distance - HP_distance) is shown. The overall probability of the activation peaks in both sub-comparisons was multiplied to calculate $P_{-}$overall. SMG $=$supramarginal gyrus, $\mathrm{PCu}=$ precuneus, IFS $=$ inferior frontal sulcus, Occ. $=$ primary visual cortex, $\mathrm{MFG}=$ middle frontal gyrus, $\mathrm{MFC}=$ medial frontal cortex, $\mathrm{mSTS}=$ middle superior temporal sulcus.

\begin{tabular}{cccc}
\hline & \multicolumn{1}{c}{ Brain Areas Responding to High-Probability Actions } & \\
\hline Area & BA & MNI $(\mathbf{x}, \mathbf{y}, \mathbf{z})$ & P_Overall \\
SMG & 48 & $-54,-42,30$ & $\mathrm{p}=0.00006$ \\
SMG & 41 & $-46,-44,26$ & $\mathrm{p}=0.00009$ \\
PCu & 7 & $0,-44,46$ & $\mathrm{p}=0.0001$ \\
IFS & 48 & $-46,20,30$ & $\mathrm{p}=0.00006$ \\
Occ. & 18,19 & $-26,-90,16$ & $\mathrm{p}=0.00003$ \\
Occ. & $17,18,19$ & $34,-84,8$ & $\mathrm{p}=0.00006$ \\
MFG & 46 & $-34,54,24$ & $\mathrm{p}=0.000096$ \\
& & Brain Areas Responding to Low-Probability Actions & $\mathbf{P}$ Overall \\
Area & BA & MNI (x, y, z) & $\mathrm{p}=0.0001$ \\
MFC & $32 / 10$ & $-14,48,12$ & $\mathrm{p}=0.000045$ \\
mSTS & 48 & $48,-8,-4$ &
\end{tabular}

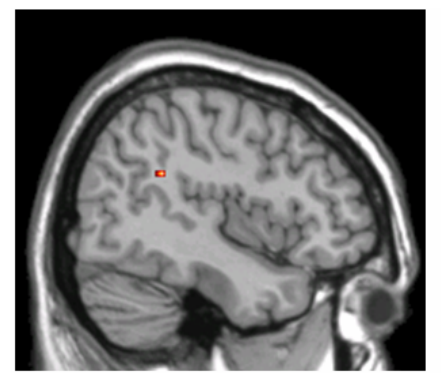

(a)

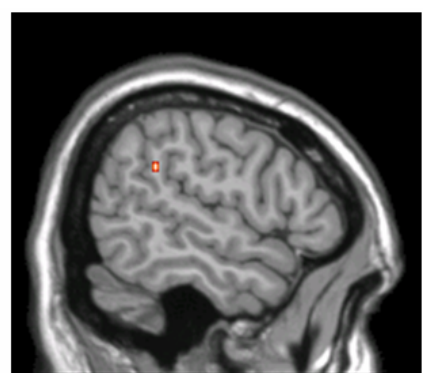

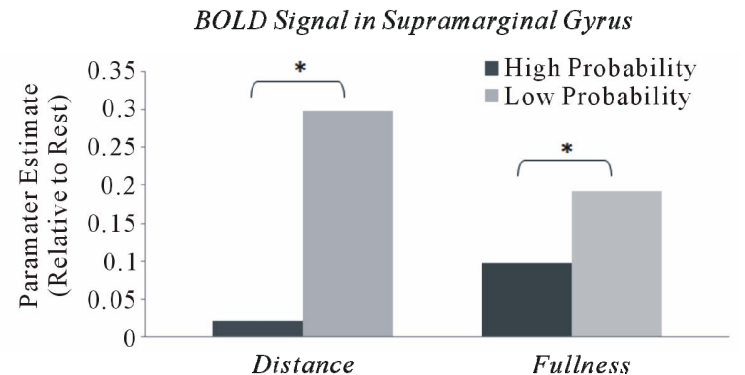

(b)

Figure 2. Brain areas coding for high-probability actions. (a) Sites of peak activation at locations in the left supramarginal gyrus (SMG) which responded more strongly to high as compared to low-probability actions, based on both fullness and distance based cues. Results are overlaid on a standard high-resolution T1 weighted brain image; (b) Graphs of BOLD signal extracted from the two sites in the left SMG and collapsed across the two sites.
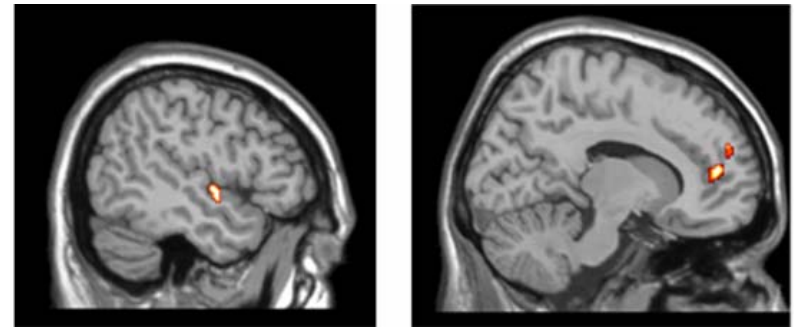

Figure 3. Brain areas coding for low-probability actions. Sites of peak activation at locations in the medial frontal cortex (MFC) and middle superior temporal sulcus (mSTS) which responded more strongly to low as compared to highprobability actions, based on both fullness and distance based cues. These sites constitute core components of the theory of mind network. Results are overlaid on a standard high-resolution $\mathrm{T} 1$ weighted brain image.

\subsection{Parametric Variations in BOLD Signal}

In order to further explore the nature of the neural response to actions of varying probability in core areas identified as differentiating between high and low probability actions we performed a secondary analysis. To examine distance based probability we recoded the fMRI data such that all actions were divided into 5 levels of probability, ranging from low to high, based on the distance traveled by the bottle (The shortest distance was coded as 1 and the longest was coded as 5). To examine fullness based probability, we recoded the fMRI data such that all actions were divided into three levels of probability (Low $=$ pouring into a glass with the same fullness as the other glass, Medium = pouring into a slightly more empty glass, High = pouring into a much more 
empty glass). We then extracted percent signal change relative to rest for all levels of probability and for all brain regions identified in Table 1 (Figure 4).

\subsection{Action Probability and Life Experience}

Finally, in order to further explore the nature of the activations observed for high and low probability actions within the MNS, we ran a correlation between the age of the participants and the strength of the differences in SMG activation observed for high and low probability actions based on either distance $\left(\mathrm{HP}_{\mathrm{d}}-\mathrm{LP}_{\mathrm{d}}\right)$ or fullness $\left(\mathrm{HP}_{\mathrm{f}}-\mathrm{LP}_{\mathrm{f}}\right)$ cues. The correlation between age and distance based differences in BOLD signal $\left(\mathrm{HP}_{\mathrm{d}}-\mathrm{LP}_{\mathrm{d}}\right)$ was significant at the more medial supramarginal site $[\mathrm{MNI}=$ $-46,-44,26](\mathrm{R}=0.44, \mathrm{p}<0.05)$, but not at the more lateral supramarginal site $[\mathrm{MNI}=-54,-42,30](\mathrm{r}=0.24$, $\mathrm{p}>0.05)$. The correlation between age and fullness based

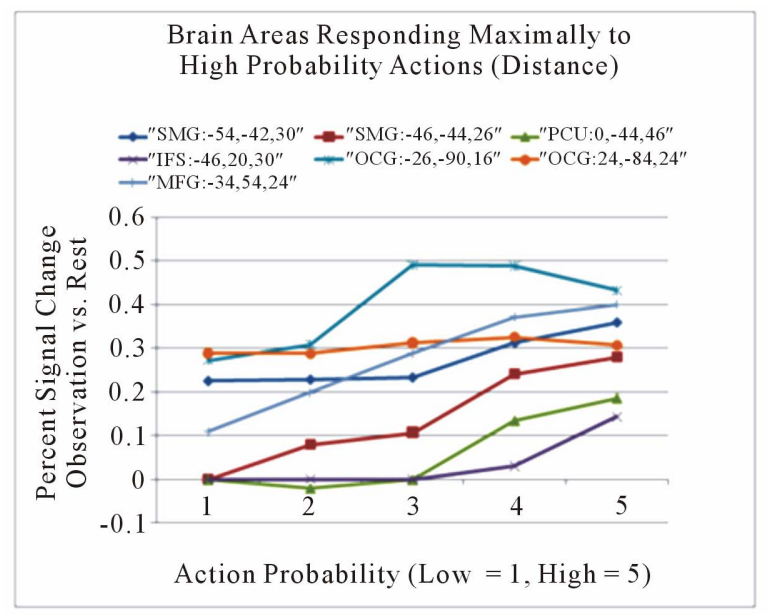

(a)

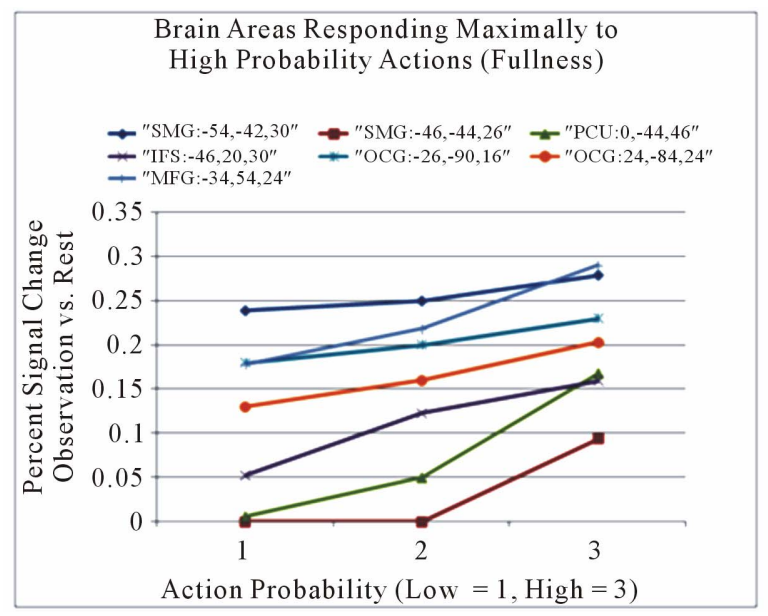

(c) differences in BOLD signal $\left(\mathrm{HP}_{\mathrm{f}}-\mathrm{LP}_{\mathrm{f}}\right)$ was significant at the more lateral site $[\mathrm{MNI}=-54,-42,30](\mathrm{R}=0.46, \mathrm{p}$ $<0.05)$, but not at the more medially situated site [MNI $=$ $-46,-44,26](\mathrm{r}=0.28, \mathrm{p}>0.05)$ (Figure 5).

\section{Discussion}

\subsection{Brain Response to Likely Actions}

Only one brain region within the putative MNS [18] differentiated between high and low-probability actions as defined in the current experiment. Two nearby, but separate sites within the left supramarginal gyrus responded maximally when there was a match between the observed and expected actions (i.e. high-probability actions). Based on both lesion and neuroimaging studies, the SMG is viewed as highly specialized neural tissue which houses visual and motor programs involved in

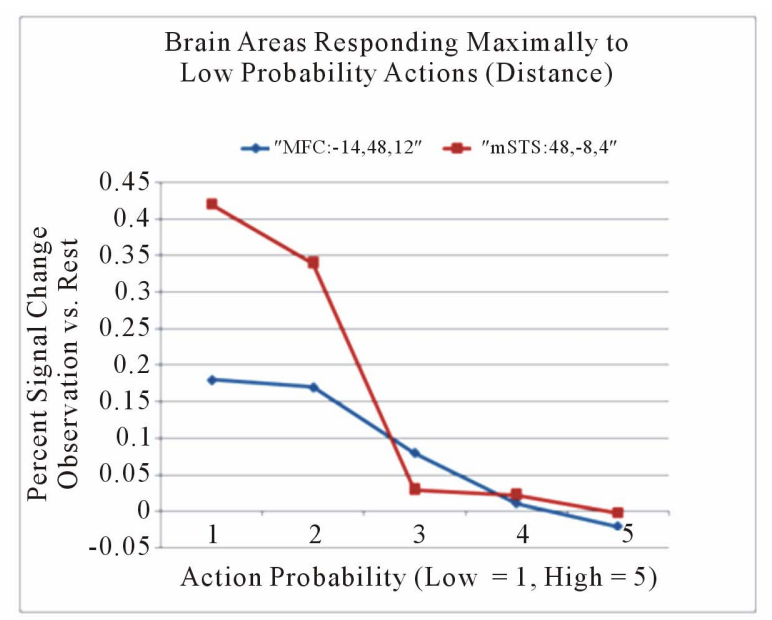

(b)

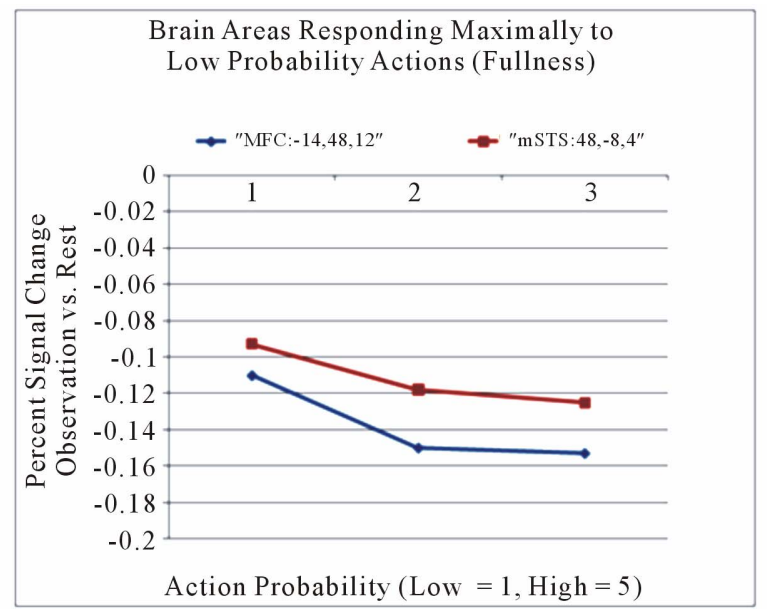

(d)

Figure 4. Plots of percent signal change elicited by observation of actions with varying degrees of probability in areas identified as having heightened responses to high (A) or low (B) probability actions (see Table 1). SMG = supramarginal gyrus, $\mathrm{PCu}=$ precuneus, IFS $=$ inferior frontal sulcus, $\mathrm{OCC} .=$ primary visual cortex, $\mathrm{MFG}=$ middle frontal gyrus, $\mathrm{MFC}=$ medial frontal cortex, $\mathrm{mSTS}=$ middle superior temporal sulcus. 

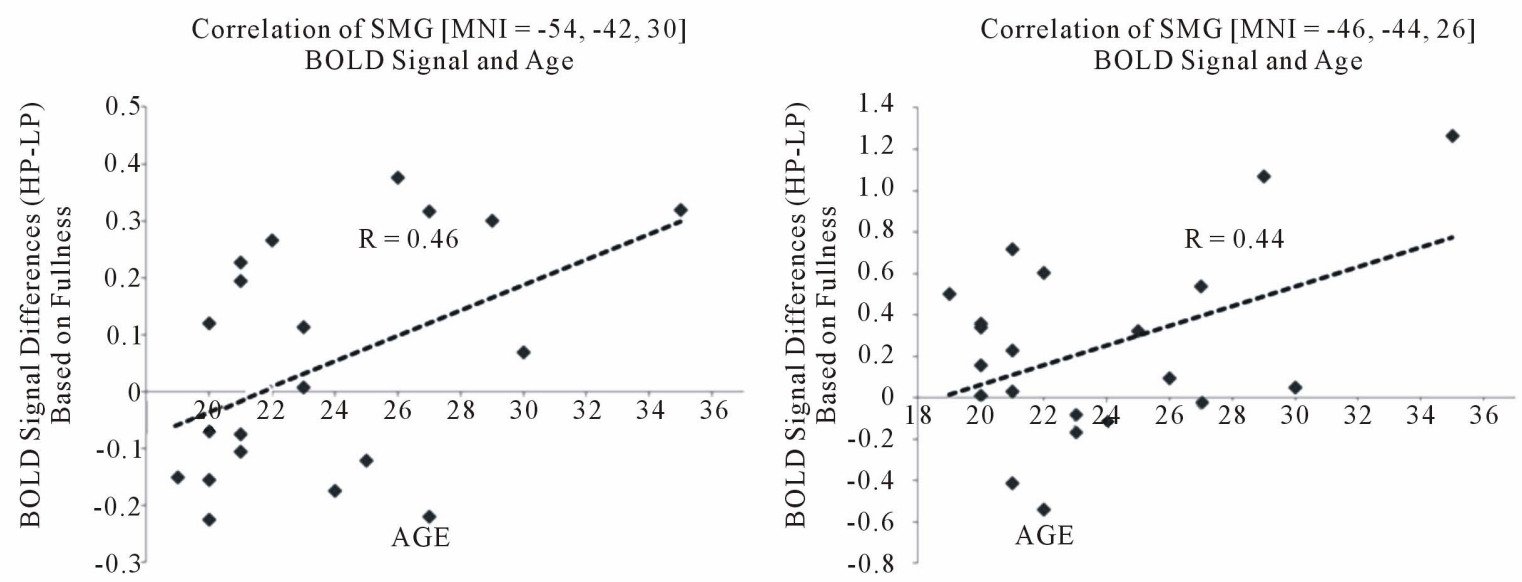

Figure 5. Correlation between age and the strength of the difference in BOLD signal elicited by high and low-probability actions (HP-LP) at sites in the supramarginal gyrus. Actions could be categorized as high or low probability based on either 1) the relative Fullness of the glass being poured into or 2) the Distance between the bottle and poured-into glass. At one site in the supramarginal gyrus $[\mathrm{MNI}=-\mathbf{5 4}, \mathbf{- 4 2 , 3 0 ]}$, the correlation between Fullness based HP-LP was significant, $(\mathrm{R}=0.46, \mathrm{p}<$ $0.05)$, but when the probability was based on Distance, the correlation was not significant $(R=0.24, p>0.05)$. At the other supramarginal site $[\mathrm{MNI}=-46,-44,26]$, the correlation between Distance based HP-LP was significant $(R=0.44, p<0.05)$, while the strength of this correlation was not significant when probability was based on Fullness.

skilled tool use [19-23]. Interestingly, sites in the MNS were found to be co-activated with the SMG in this and other experiments, suggesting a close functional relationship with each other [24,25]. The current data suggest an important role for the left SMG in differentiating between actions of low and high probability. Adopting a probabilistic perspective allows us to look at skilled tool use in a new way. Tool use is made possible by the ability to infer future actions specific to that tool. Such inferences are especially important to tool/object use because proper handling of these items is typically associated with complex sequences of actions which must be executed in the appropriate order. For example, a mallet implies a series of actions associated with pounding in or removing a nail.

It may be interesting to consider how certain brain sites, such as the SMG, come to respond differently to actions of either high or low probabilities. The simplest and most parsimonious explanation is that probabilities are, as implied by Bayes' Rule, based on prior experience or entrainment. In the case of the current experiment, participants' personal experience in social drinking may have led to the formation of specific expectations regarding the relationship between the relative fullness of two glasses and subsequent pouring actions. The relationship between inter-object distance and probability, on the other hand, may be rooted in the more general experience of preferring the combination of proximate objects across a variety of contexts, something commonly referred to as the Gestalt principle of Proximity (e.g. we might eat the closest French fry off our plate first, the most distant last). The present research indicates a common neural substrate for evaluating action probabilities based on both types of cues (fullness and distance).

An interesting finding in the current experiment is that we did observe a correlation between age, which is representative of a participant's general life experience, the strength of the probability coding at sites in the supra marginal gyrus. It seems that the two sites within the left supramarginal gyrus, while both responding more strongly to high-probability actions, were differentially sensitive whether or not the likelihood of an action was related to the proximity of objects being combined or the relative need to refill a certain glass. First, this finding makes it less likely that the supramarginal activations uncovered in the conjunction analysis are the result of insufficient thresholding (i.e. Type I errors). Second, this finding seems to support the idea that the supramarginal gyrus's response to action probabilities is, at least in part, governed by prior experience (either amount or type). This finding also raises the interesting possibility that different sub-areas within the parietal lobe may code for probability of actions based on different types of cues (e.g. fullness or distance based). Of course this claim is highly speculative and further experimentation is needed to confirm this hypothesis. More sensitive questionnaires, perhaps dealing with specific types of motor experience (which would not rely on assuming on the link between age and specific types of experiences), could be used in future studies which examine patterns of neural activity elicited by high and low-probability actions.

Finally, we would like to note that the precuneus and primary visual cortices showed the same pattern of responses as the SMG during observation of probable and 
improbable actions, although these activity patterns were not correlated with participants' age. The precuneus is thought to be involved in visuo-spatial imagery (as is primary visual cortex) and episodic memory retrieval [26, 27]. The co-activation of these areas during the observation of high-probability actions suggests a relationship between past experience, upon which action probabilities are probably built and mental imagery, which could be used to replay or simulate previously observed actions. The precise nature of the relationship between learning, mental imagery and probability coding requires further investigation.

\subsection{Brain Response to Unlikely Actions}

During observation of low probability actions, sites commonly associated with evaluating the intentions of others, including the medial frontal cortex and the superior temporal sulcus, were found to be active. The MFC is a core component of the theory of mind (ToM) network, a network found to be active when people evaluate about or consider the intentions of other people. Based on numerous studies reporting co-activation of STS and MFC [28-31], as well as the known anatomical connectivity between these two areas [32], co-activation in these two areas has been hypothesized to reflect core mentalizing processes [33]. The current data are consistent with this interpretation. As such, we take this activity in the MFC and $\mathrm{mSTS}$ to represent subjects' attempt to understand the intentions behind the actor's improbable movement choice. Also informative is our failure to find MNS involvement during the observation of low-probability actions. Indeed, the exact role of the MNS in the processing of atypical, or odd actions, is currently under debate. Some evidence suggests that humans rely on their own motor systems to make sense of others' actions, and to recognize their intentions $[11,14,18,34]$, while other authors have failed to observe additional MNS activation during the observation of atypical actions [13].

\section{Summary and Conclusions}

Results from the present experiment are consistent with the idea that the human brain differentiates between actions of differing probability insofar as the functional signatures of probable and improbable actions can be differentiated using modern neuroscience techniques. As such, these data take an important step towards validating mathematical models of psychological phenomenon which incorporate probabilistic equations.

The current results also raise a number of interesting questions regarding the neural underpinnings of the highly sophisticated human apacity for estimating action likelihoods. For example, how exactly do specific brain areas become entrained to differentiate high and low probabil- ity actions? And does our ability to estimate an action's likelihood emerge differently depending on the specific types of cues we use to arrive at our estimates? Perhaps most interestingly, what sorts of computational models of mirror neurons might account for the responses observed in euroimaging experiments involving observation of usual and unusual actions? Besides obvious implications for researchers studying action/intention recognition, social-motor control and joint action, it is very likely that a better understanding of the neural mechanisms that support the human capacity for action prediction could lead to radical improvements in the quality of human-human and human-computer interactions.

\section{Acknowledgements}

The present research was supported by the EU-project Joint Action Science and Technology (JAST) (IST-FP6003747).

\section{REFERENCES}

[1] H. R. Heekeren, S. Marrett and L. G. Ungerleider, "The Neural Systems That Mediate Human Perceptual Decision Making," Nature Reviews Neuroscience, Vol. 9, No. 6, 2008, pp. 467-479. doi:10.1038/nrn2374

[2] R. H. Cuijpers, et al., "Goals and Means in Action Observation: A Computational Approach," Neural Networks, Vol. 19, No. 3, 2006, pp. 311-322. doi:10.1016/j.neunet.2006.02.004

[3] M. Haruno, D. M. Wolpert and M. Kawato, "MOSAIC Model for Sensorimotor Learning and Control," Neural Computation, Vol. 13, No. 10, 2001, pp. 2201-2220. doi:10.1162/089976601750541778

[4] E. Oztop, D. Wolpert and M. Kawato, "Mental State Inference Using Visual Control Parameters," Cognitive Brain Research, Vol. 22, No. 2, 2005, pp. 129-151. doi:10.1016/j.cogbrainres.2004.08.004

[5] D. M. Wolpert, "Probabilistic Models in Human Sensorimotor Control," Human Movement Science, Vol. 26, No. 4, 2007, pp. 511-524. doi:10.1016/j.humov.2007.05.005

[6] J. I. Gold and M. N. Shadlen, "The Neural Basis of Decision Making," Annual Review of Neuroscience, Vol. 30, 2007, pp. 535-574.

doi:10.1146/annurev.neuro.29.051605.113038

[7] T. Yang and M. N. Shadlen, "Probabilistic Reasoning by Neurons," Nature, Vol. 447, No. 7148, 2007, pp. 10751080. doi: $10.1038 /$ nature 05852

[8] K. Preuschoff, S. R. Quartz and P. P. Bossaerts, "Human Insula Activation Reflects Risk Prediction Errors as Well as Risk," Journal of Neuroscience, Vol. 28, No. 1, 2008, pp. 2745-2752. doi:10.1523/JNEUROSCI.4286-07.2008

[9] K. Samejima, et al., "Representation of Action-Specific Reward Values in the Striatum," Science, Vol. 310, No. 5752, 2005, pp. 1337-1340. doi:10.1126/science. 1115270

[10] B. Calvo-Merino, et al., "Seeing or Doing? Influence of 
Visual and Motor Familiarity in Action Observation," Current Biology, Vol. 16, No. 19, 2006, pp. 1905-1910. doi:10.1016/j.cub.2006.07.065

[11] T. Koelewijn, et al., "Motor-Cortical Beta Oscillations Are Modulated by Correctness of Observed Action," Neuroimage, Vol. 20, No. 2, 2008, pp. 767-775. doi:10.1016/j.neuroimage.2007.12.018

[12] G. Buccino, G. R. Fink, N. J. Shah, K. Zilles, R. J. Seitz and H. J. Freund, "Observation and Imitation of Object Related Actions,” Neuroimage, Vol. 13, No. 5, 2001.

[13] F. P. de Lange, et al., "Complementary Systems for Understanding Action Intentions," Current Biology, Vol. 18, No. 6, 2008, pp. 454-457. doi:10.1016/j.cub.2008.02.057

[14] L. Fogassi, et al., "Parietal Lobe: From Action Organization to Intention Understanding," Science, Vol. 308, No. 5722, 2005, pp. 662-667. doi:10.1126/science. 1106138

[15] M. Iacoboni, et al., "Grasping the Intentions of Others with One's Own Mirror Neuron System," PLoS Biology, Vol. 3, No. 3, 2005, p. e79. doi:10.1371/journal.pbio.0030079

[16] G. Metta, G. Sandini, L. Natale, L. Craighero and L. Fadiga, "Understanding Mirror Neurons: A Bio-Robotic Approach," Interaction Studies, Vol. 7, No. 2, 2006, pp. 197-232. doi:10.1075/is.7.2.06met

[17] M. Iacoboni, "Perspectives on Imitation: From Mirror Neurons to Memes," MIT Press, Cambridge, 2005, pp. 77-100.

[18] G. Rizzolatti and L. Craighero, "The Mirror-Neuron System," Annual Review of Neuroscience, Vol. 27, No. 1, 2004, pp. 169-192. doi:10.1146/annurev.neuro.27.070203.144230

[19] E. A. Roy, et al., "Analysis of Task Demands in Apraxia," International Journal of Neuroscience, Vol. 56, No. 1-4, 1991, pp. 177-186. doi:10.3109/00207459108985414

[20] N. Geschwind and E. Kaplan, "A Human Cerebral Deconnection Syndrome: A Preliminary Report," Neurology, Vol. 12, No. 10, 1962, pp. 675-685. doi:10.1212/WNL.12.10.675

[21] G. Goldenberg, "Pantomime of Object Use: A Challenge to Cerebral Localization of Cognitive Function," Neuroimage, Vol. 20, No. 1, 2003, pp. S101-S106. doi:10.1016/i.neuroimage.2003.09.006

[22] G. Goldenberg, K. Hartmann and I. Schlott, "Defective Pantomime of Object Use in Left Brain Damage: Apraxia or Asymbolia?" Neuropsychologia, Vol. 41, No. 12, 2003, pp. 1565-1573. doi:10.1016/S0028-3932(03)00120-9

[23] S. H. Johnson-Frey, R. Newman-Norlund and S. T. Grafton, "A Distributed Left Hemisphere Network Active during Planning of Everyday Tool Use Skills," Cereb Cortex,
Vol. 15, No. 6, 2005, pp. 681-695. doi:10.1093/cercor/bhh169

[24] G. Buccino, et al., "Neural Circuits Underlying Imitation Learning of Hand Actions: An Event-Related fMRI Study," Neuron, Vol. 42, No. 2, 2004, pp. 323-334. doi:10.1016/S0896-6273(04)00181-3

[25] P. D. McGeoch, D. Brang and V. S. Ramachandran, “Apraxia, Metaphor and Mirror Neurons," Medical Hypotheses, Vol. 69, No. 6, 2007, pp. 1165-1168. doi:10.1016/j.mehy.2007.05.017

[26] A. E. Cavanna and M. R. Trimble, "The Precuneus: A Review of Its Functional Anatomy and Behavioral Correlates," Brain, Vol. 129, No. 3, 2006, pp. 564-583. doi:10.1093/brain/awl004

[27] S. M. Kosslyn, G. Ganis and W. L. Thompson, "Neural Foundations of Imagery," Nature Reviews Neuroscience, Vol. 2, No. 9, 2001, pp. 635-642. doi:10.1038/35090055

[28] H. Takahashi, N. Yahata, M. Koeda, T. Matsuda, K. Asai and Y. Okubo, "Brain Activation Associated with Evaluative Processes of Guild and Embarrassment: An fMRI Study," Neuroimage, Vol. 23, No. 3, 2004, pp. 967-974. doi:10.1016/j.neuroimage.2004.07.054

[29] C. Kobayashi, G. H. Glover and T. Elise, "Children's and Adults' Neural Bases of Verbal and Nonverbal 'Theory of Mind'," Neuropsychologia, Vol. 45, No. 7, 2007, pp. 1522-1532. doi:10.1016/j.neuropsychologia.2006.11.017

[30] B. A. Voellm, A. N. W. Taylor, P. Richardson, R. Corcoran, J. Stirling, S. McKie, J. F. Deakin and R. Elliot, "Neuronal Correlates of Theory of Mind and Empathy: A Functional Magnetic Resonance Imaging Study in a Nonverbal Task," Neuroimage, Vol. 29, No. 1, 2006, pp. 9098. doi:10.1016/j.neuroimage.2005.07.022

[31] H. L. Gallagher, F. Happe, N. Brunswick, P. C. Fletcher, U. Frith and C. D. Frith, "Reading the Mind in Cartoons and Stories: An fMRI Study of 'Theory of Mind' in Verbal and Nonverbal Tasks," Neuropsychologia, Vol. 38, No. 1, 2000, pp. 11-21. doi:10.1016/S0028-3932(99)00053-6

[32] B. Seltzer and D. N. Pandya, "Frontal Lobe Connections of the Superior Temporal Sulcus in the Rhesus Monkey," Journal of Comparative Neurology, Vol. 281, No. 1, 1989, pp. 97-113. doi:10.1002/cne.902810108

[33] G. Hein and R. T. Knight, "Superior Temporal SulcusIt's My Area: Or Is It?" Journal of Cognitive Neuroscience, Vol. 20, No. 12, 2008, pp. 1-12. doi:10.1162/jocn.2008.20148

[34] V. Gallese and A. Goldman, "Mirror Neurons and the Simulation Theory of Mind-Reading," Trends in Cognitive Sciences, Vol. 2, No. 12, 1998, pp. 493-501. doi:10.1016/S1364-6613(98)01262-5 